\title{
AGROECOLOGÍA EN LA ESCUELA Y CALIDAD DE VIDA RELACIONADA A LA SALUD DE LOS ADOLESCENTES
}

\author{
SCHOOL AGROECOLOGY AND HEALTH-RELATED QUALITY OF LIFE TO ADOLESCENTS \\ Daniela Moyano ${ }^{1,2}$, María Cecilia Scándolo ${ }^{3}$, Nilda Raquel Perovic 4.
}

\begin{abstract}
Resumen:
El estudio tuvo como objetivo evaluar la contribución de la agroecología escolar a la calidad de vida relacionada a la salud de los adolescentes de una escuela de Córdoba, Argentina. Estudio de factibilidad con diseño cuasi experimental y con grupo control llevado a cabo en una escuela pública de nivel medio. La muestra fue de 58 adolescentes en el grupo intervención y 77 en el grupo control.

Después de la intervención se observó un incremento significativo en el consumo de alimentos agroecológicos (de un $26 \%$ a un $74 \%$ p $<0,05$ ). Los conocimientos teóricos-prácticos adquiridos sobre agroecología a nivel áulico aumentaron de un 37\% a un 63\% ( $p<0,05)$. Se detectaron cambios significativos en la sensibilización con el ambiente y en la dimensión de relacionamiento $(p<0,05)$.

Se concluye que implementar agroecología escolar puede brindar aportes resolutivos a algunas de las problemáticas de la calidad de vida relacionada a la salud de adolescentes.
\end{abstract}

Palabras clave: adolescentes; escuela; calidad de vida; agroecología.

\section{Abstract:}

The study aimed to evaluate the contribution of school agroecology to the quality of life related to health of adolescents of a school of Cordoba, Argentina. Feasibility study. It was a study quasi- experimental whit control group carried out in a middle-level public school. The sample consisted of 58 adolescents in the intervention group and 77 in the control group.

After the intervention increased consumption of agro-ecological food (from $26 \%$ to $74 \% p<0,05$ ). Changes were detected in acquired knowledge about agroecology in the classroom level going from $37 \%$ to $63 \%(p<0,05)$. Positive changes detected in awareness with the environment and relationship $(p<0,05)$.

It was evidenced that the implementation of school agroecology can provide resolutive contributions to some of the problems the health related quality of life of adolescents.

Keywords: adolescents; school; quality of life; agroecology.

1 Licenciada en Nutrición. Magíster en Salud Pública. Escuela de Nutrición, Facultad de Ciencias Médicas, Universidad Nacional de Córdoba, Argentina

2 Email de contacto: moyanodaniela12@gmail.com

3 Licenciada en Nutrición. Escuela de Nutrición, Facultad de Ciencias Médicas, Universidad Nacional de Córdoba, Argentina

4 Licenciada en Nutrición. Doctora en Ciencias de la Salud. Escuela de Nutrición, Facultad de Ciencias Médicas, Universidad Nacional de Córdoba, Argentina

Recibido: 2017-08-07 Aceptado: 2017-10-03

DOI: 10.31053/1853.0605.v75.n2.17560 


\section{Introducción}

En las últimas décadas han surgido reconocimientos de los derechos de niños, niñas y adolescentes que marcaron el comienzo de transformaciones en torno a las prácticas, instituciones y políticas destinadas a estos grupos, reconociéndolos como sujetos titulares activos de derechos.

Si bien se observaron mejoras en algunos aspectos de la calidad de vida de los adolescentes la evidencia actual pone en relieve diversas problemáticas que afectan negativamente la calidad de vida y que repercuten en su desarrollo sano y armónico y llevando a vulnerar sus derechos ${ }^{(1-4)}$. En Argentina la población de niños, niñas y adolescentes ( 0 a 17 años) representa un 30,7\%(2) donde el 10,3\% de este grupo se encuentra bajo la línea de pobreza ${ }^{(3)}$.

Hablar de calidad de vida en la adolescencia consiste en pensarla como "proceso" y "estado", desde un enfoque multidimensional, rescatando tanto condiciones objetivas como subjetivas ${ }^{(4)}$, en este contexto la escuela se constituye en un ambiente estratégico.

Se define a la agroecología como una disciplina científica, un conjunto de prácticas y un movimiento social que permitirá lograr ciertas trasformaciones a nivel de la escuela en el que se desenvuelven los alumnos (FAO, 2014).

La agroecología escolar se considera una de las estrategias socio-pedagógicas con mayor impacto y potencial para lograr abordajes complejos generando canales participativos, inclusivos y de intercambios de experiencias, conocimientos y saberes tanto dentro como fuera del aula (FAO, 2014), donde los adolescentes se convierten en protagonistas principales en un marco de un proceso de aprendizaje significativo ${ }^{(5)}$.

Algunos experiencias recientes reportaron que la agroecología escolar promueve el consumo de alimentos saludables y espacios de educación nutricional. Además permitiría mejorar algunas situaciones de salud poco satisfactorias, como es la prevalencia de obesidad y otros factores de riesgo ${ }^{(6-}$ 16).

Otros antecedentes mostraron que puede actuar sobre algunas dimensiones subjetivas y objetivas de la calidad de vida como es la educación, el ambiente, aspectos subjetivos individuales y colectivos siendo elementos claves al momento de definir los procesos de salud-enfermedad durante la etapa de la adolescencia $^{(5,17-22)}$.

La escasa evidencia documentada en el campo explorado en el país y la región da lugar a poner en relieve la importancia de producir evidencia sobre el impacto de las intervenciones "en el mundo real" que emergen desde escuela y que buscan contribuir a la calidad de vida de los adolescentes desde una perspectiva integral.

El objetivo principal de este estudio fue evaluar la contribución de la implementación de agroecología escolar a la calidad de vida relacionada a la salud de los adolescentes que asisten a una escuela pública de la ciudad de Córdoba, Argentina en el periodo 2015-2016.

\section{Material y Métodos}

\section{Tipo de estudio}

Fue un estudio de factibilidad que formó parte de una iniciativa intersectorial que dio origen a un proyecto de intervención llevado a cabo durante el periodo 2015-2016 a partir de alianzas entre diferentes sectores públicos en el marco de sumar acciones a la iniciativa de "Escuelas Sostenibles" propuesta por la Organización para las Naciones Unidas para la Alimentación y Agricultura (FAO).

El componente de investigación tuvo un diseño cuasi experimental con un grupo control. La unidad de análisis consistió en una escuela secundaria pública urbana de la ciudad de Córdoba, Argentina seleccionada a través de muestreo intencional en base a criterios de vulnerabilidad social del contexto en el cual se inserta(2).

\section{Participantes}

Se invitaron a participar a alumnos que asistían a una escuela pública de nivel medio previamente seleccionada y donde cumplían con los siguientes criterios de inclusión: pertenecer/o haber pertenecido 
durante los últimos 3 años a la escuela seleccionada, estar transitando la adolescencia/juventud (entre 10 y 24 años), residir dentro de mismo contexto (barrio) donde se inserta la escuela.

Dado que fue un estudio de factibilidad donde no se requirió de aplicar el cálculo de tamaño muestral, en este estudio se decidió incluir al grupo de intervención (GI) a todos los alumnos de ambos géneros asistentes a los últimos 3 años del sistema de educación formal (4운 $5^{\circ}$ y $\left.6^{\circ}\right)$ y al grupo control (GC) a

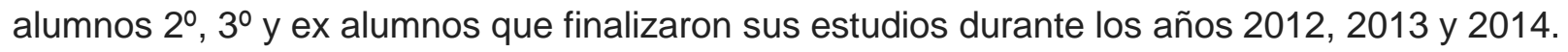

Para la recolección de datos de calidad de vida relacionada a la salud (CVRS) de los adolescentes se implementó un instrumento de encuesta semi-estructurada auto-administrado (preguntas cerradas, abiertas y mixtas) (Anexo. 1). Para los aspectos subjetivos se tomaron algunos indicadores del instrumento validado Comprehensive Quality of Life Scale, School Version ${ }^{(23)}$ y el diseño global del instrumento respondió a las dimensiones Materiales, Ambientales y de Relacionamiento ${ }^{(28,29)}$ consideradas principales determinantes de la salud.

\section{Intervención escolar}

El periodo de intervención en el grupo de adolescentes fue durante 12 meses (con mediciones basales y a los 12 meses). Las actividades programadas se ejecutaron en el marco del programa de los espacios curriculares de ciencias naturales, a partir de acuerdos formales con la institución.

La metodología de planificación y ejecución de las actividades durante la intervención fue basada en el modelo metodológico Comunidad de Aprendizaje $(\mathrm{CA})^{(5)}$ que tiene como eje principal al adolescente como protagonista partiendo de un fuerte trabajo previo sobre las concepciones espontáneas y desde un aprendizaje significativo ${ }^{(24,25)}$, desde donde se abordaron las diferentes dimensiones de la CVRS impulsadas desde la escuela (Figura. 1).

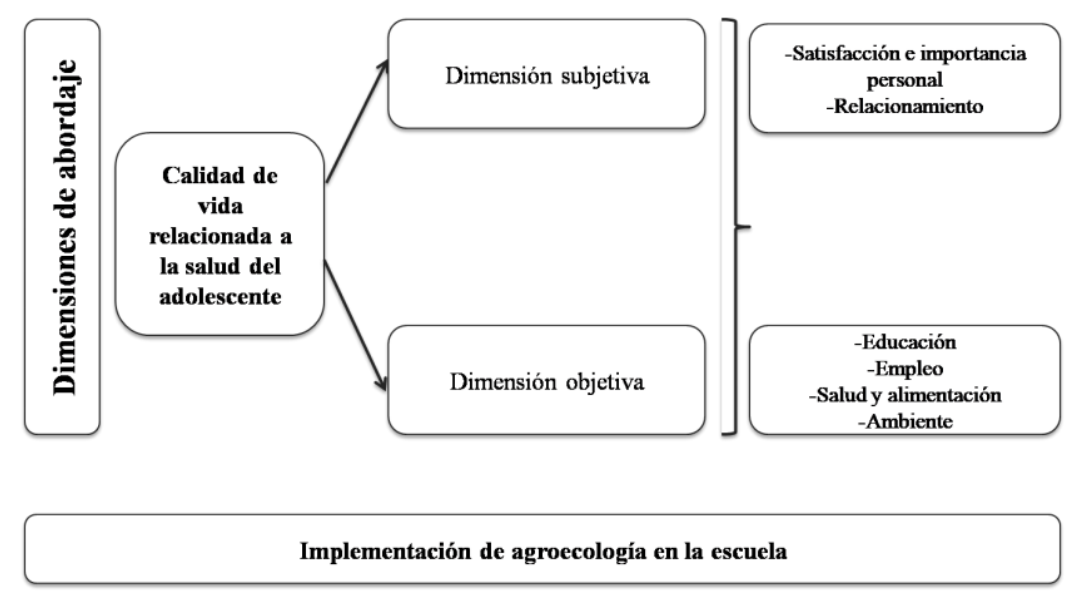

Figura 1. Framework de implementación de la intervención

Se tuvieron en cuenta los siguientes aspectos: construir sobre procesos ya en marcha/adolescentes como destinatarios y actores principales/procesos participativos en el diseño, ejecución y evaluación del plan de trabajo/proyecto asociativo y construcción de alianzas/orientación hacia el aprendizaje y énfasis sobre la innovación pedagógica/revitalización y renovación del sistema de educativo público/prioridad sobre el adolescente y su desarrollo integral/intervención sistémica y búsqueda de articulaciones/evaluación, sistematización y difusión de la experiencia/continuidad y sustentabilidad de los esfuerzos.

El planteo de las actividades fue con modalidad de talleres teórico-prácticos o seminarios participativos donde se intervino en las dimensiones Materiales, Ambientales y Relacionales de la calidad de vida relacionada a la salud de los y las adolescentes a través de la estrategia seleccionada (agroecología escolar).

Las actividades fueron: talleres participativos sobre implementación y seguimiento de huertas ecológicas, cine debate ambiental con enfoque agro-productivo, cocina ecológica, alimentación saludable, laboratorio para análisis de contaminación ambiental; implementación de contenidos de agroecología vinculados a diferentes dimensiones de la CVRS en las materias de la currícula escolar; talleres de capacitación y sensibilización sobre micro-emprendimientos alimentarios. Las actividades se 
llevaron a cabo en el marco de la escuela como también en diferentes espacios comunitarios, siempre que fue posible y con presencia de los docentes y/o otros actores escolares durante el desarrollo de las actividades. Se contó con diferentes herramientas y materiales didácticos de apoyo a medida que se desarrollen las temáticas.

\section{Educación habitual}

Los adolescentes que formaron parte del grupo control siguieron su plan curricular habitual y no recibieron la intervención a partir de la estrategia de agroecología. A este grupo se le realizó las mediciones basales y a los 12 meses de igual manera que en el grupo intervención.

\section{Análisis estadístico}

Se realizó un análisis estadístico descriptivo univariado utilizando medidas de posición y dispersión. Se recurrió a la prueba T de Student, ANAVA, Kruskal Wallis y un análisis bivariado a través de la prueba de $x 2(p<0,05)$; se utilizo el software estadístico Infostat.

\section{Resultados}

\section{Indicadores de implementación}

Durante los 12 meses de intervención se cumplieron el 100\% de las actividades planificadas. El 15\% en $4^{\circ}, 27 \%$ en $5^{\circ}$ y $28 \%$ en $6^{\circ}$ año del total de los espacios curriculares destinaron horas cátedras a la implementación de las actividades planificadas.

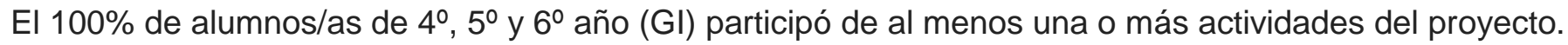
El $32 \%$ del total de docentes de los cursos bajo estudio participó de al menos una actividad y el $49 \%$ de los adolescentes del GI realizó una huerta ecológica en su hogar.

El 94\% de los participantes de la intervención refirieron un nivel de satisfacción entre "aceptable" "Bueno" o "muy bueno".

\section{Resultados primarios}

Al inicio (pre-test) de la intervención participaron 136 adolescentes; 58 en el Gl y 77 en el GC; y al finalizar (pos-test) fueron 100 adolescentes (43 en Gl y 57 en GC), representada por un $43 \%$ de hombres y un $57 \%$ de mujeres. La tasa de perdida durante el seguimiento de la intervención fue de $26 \%$ individuos, donde las razones de esta perdida en el Gl fue por deserción escolar; mientras que en el GC se debió a la deserción y ausentismo durante el relevamiento de la instancia pos-test.

Un $72 \%$ de adolescentes del Gl tuvo entre 14 a 17 años y el $28 \%$ fue mayor a 17 años. La media de edad en mujeres fue inferior a la de los varones $(16,64$ vs. 17,39$)(p<0,05)$. Mientras que la media de edad del GC fue 15,5 años.

Se observaron diferencias estadísticamente significativas ( $p<0,05$ con un IC del $95 \%$ ) entre la etapa pretest y pos-test en el GI en las diferentes dimensiones de la calidad de vida relacionada a la salud (Tabla 1).

Tabla 1. Análisis del pre-test y post-test en las dimensiones de la CVRS del grupo intervención

\begin{tabular}{|c|c|c|c|c|c|c|c|c|}
\hline \multirow{2}{*}{\multicolumn{2}{|c|}{$\begin{array}{l}\text { Dimensiones de CVRS } \\
\text { Educación }\end{array}$}} & \multicolumn{2}{|c|}{ Pre-test } & \multicolumn{2}{|c|}{ Pos-test } & \multicolumn{2}{|c|}{ Total } & \multirow[b]{2}{*}{$\mathbf{p}$} \\
\hline & & $\mathbf{N}$ & $\%$ & $\mathbf{N}$ & $\%$ & $\mathbf{N}$ & $\%$ & \\
\hline \multirow{3}{*}{$\begin{array}{l}\text { Conocimientos teórico/práctico sobre } \\
\text { agroecología* }\end{array}$} & No & 34 & 87 & 5 & 13 & 39 & 100 & \multirow{4}{*}{0,0001} \\
\hline & Si & 23 & 37 & 39 & 63 & 62 & 100 & \\
\hline & Total & 57 & 56 & 44 & 44 & 101 & 100 & \\
\hline \multirow{3}{*}{$\begin{array}{l}\text { Conocimientos teórico/práctico adquiridos en el } \\
\text { último año sobre agroecología y ambiente* }\end{array}$} & No & 47 & 84 & 9 & 16 & 56 & 100 & \\
\hline & $S i$ & 11 & 24 & 35 & 76 & 46 & 100 & \multirow[t]{2}{*}{0,0001} \\
\hline & Total & 58 & 57 & 44 & 43 & 101 & 100 & \\
\hline \multirow{2}{*}{$\begin{array}{l}\text { Conocimientos teórico/práctico adquiridos en el } \\
\text { último año sobre agroecología y mercado de }\end{array}$} & No & 53 & 84 & 10 & 16 & 63 & 100 & \multirow[b]{2}{*}{0,0001} \\
\hline & Si & 5 & 13 & 33 & 87 & 38 & 100 & \\
\hline
\end{tabular}




\begin{tabular}{|c|c|c|c|c|c|c|c|c|}
\hline trabajo* & Total & 58 & 57 & 43 & 43 & 101 & 100 & \\
\hline \multirow{3}{*}{$\begin{array}{l}\text { Conocimientos teórico/práctico adquiridos en el } \\
\text { último año sobre agroecología y consumo de } \\
\text { alimentos* }\end{array}$} & No & 44 & 83 & 9 & 17 & 53 & 100 & \multirow{3}{*}{0,0001} \\
\hline & $S i$ & 14 & 29 & 34 & 71 & 48 & 100 & \\
\hline & Total & 58 & 57 & 43 & 43 & 101 & 100 & \\
\hline \multirow{3}{*}{$\begin{array}{l}\text { Conocimientos teórico/práctico adquiridos en el } \\
\text { último año sobre agroecología y } \\
\text { nutrición/salud* }\end{array}$} & & 39 & 85 & 7 & 15 & 46 & 100 & \multirow{3}{*}{0,0001} \\
\hline & $S i$ & 17 & 32 & 36 & 68 & 53 & 100 & \\
\hline & Total & 56 & 57 & 43 & 43 & 99 & 100 & \\
\hline \multicolumn{2}{|l|}{ Inserción laboral } & $\mathbf{N}$ & $\%$ & $\mathbf{N}$ & $\%$ & $\mathbf{N}$ & $\%$ & p \\
\hline \multirow{3}{*}{$\begin{array}{l}\text { Realización micro-emprendimiento con enfoque } \\
\text { agroecológico }\end{array}$} & $S i$ & 39 & 67 & 19 & 33 & 58 & 100 & \multirow{3}{*}{0,01} \\
\hline & No & 18 & 42 & 25 & 58 & 43 & 100 & \\
\hline & Total & 57 & 56 & 44 & 44 & 101 & 100 & \\
\hline \multicolumn{2}{|l|}{ Alimentación saludable } & $\mathbf{N}$ & $\%$ & $\mathbf{N}$ & $\%$ & $\mathbf{N}$ & $\%$ & $\mathbf{p}$ \\
\hline \multirow{3}{*}{$\begin{array}{l}\text { Conocimientos sobre alimentos } \\
\text { agroecológicos* }\end{array}$} & No & 48 & 92 & 4 & 8 & 52 & 100 & \multirow{3}{*}{0,0001} \\
\hline & $S i$ & 8 & 17 & 40 & 83 & 48 & 100 & \\
\hline & Total & 56 & 56 & 44 & 44 & 100 & 100 & \\
\hline \multirow{3}{*}{$\begin{array}{l}\text { Creencias sobre beneficios de alimentos } \\
\text { agroecológicos* }\end{array}$} & No & 34 & 89 & 4 & 11 & 38 & 100 & \multirow{3}{*}{0,0001} \\
\hline & $S i$ & 7 & 15 & 39 & 85 & 46 & 100 & \\
\hline & Total & 41 & 49 & 43 & 51 & 84 & 100 & \\
\hline \multirow{3}{*}{$\begin{array}{l}\text { Incorporación a la alimentación de nuevos } \\
\text { productos de origen vegetal (frutas y verduras) } \\
\text { en el último año }\end{array}$} & No & 21 & 66 & 11 & 34 & 32 & 100 & \multirow{3}{*}{0,16} \\
\hline & $S i$ & 34 & 51 & 33 & 49 & 67 & 100 & \\
\hline & Total & 55 & 56 & 44 & 44 & 99 & 100 & \\
\hline \multirow{3}{*}{$\begin{array}{l}\text { Consumió alguna vez verduras y/o frutas } \\
\text { agroecológicas* }\end{array}$} & No & 32 & 76 & 10 & 24 & 42 & 100 & \multirow{3}{*}{0,0001} \\
\hline & $S i$ & 12 & 26 & 34 & 74 & 46 & 100 & \\
\hline & Total & 44 & 50 & 44 & 50 & 88 & 100 & \\
\hline \multicolumn{2}{|l|}{ Ambiente } & $\mathbf{N}$ & $\%$ & $\mathbf{N}$ & $\%$ & $\mathbf{N}$ & $\%$ & $\mathbf{p}$ \\
\hline \multirow{3}{*}{$\begin{array}{l}\text { Creencias sobre relación entre producción de } \\
\text { alimentos y cuidado del ambiente* }\end{array}$} & No & 36 & 77 & 11 & 23 & 47 & 100 & \multirow{3}{*}{$\mathbf{0 , 0 0 0 1}$} \\
\hline & $S i$ & 15 & 32 & 32 & 68 & 47 & 100 & \\
\hline & Total & 51 & 54 & 43 & 46 & 94 & 100 & \\
\hline \multirow{3}{*}{$\begin{array}{l}\text { Creencias sobre influencia de factores } \\
\text { ambientales en la salud y calidad de vida }\end{array}$} & No & 9 & 69 & 4 & 31 & 13 & 100 & \multirow{3}{*}{0,29} \\
\hline & $S i$ & 45 & 54 & 39 & 46 & 84 & 100 & \\
\hline & Total & 54 & 56 & 43 & 44 & 97 & 100 & \\
\hline \multirow{3}{*}{$\begin{array}{l}\text { Realización de actividades de cuidado del } \\
\text { ambiente }\end{array}$} & No & 9 & 82 & 2 & 18 & 11 & 100 & \multirow{3}{*}{0,06} \\
\hline & $S i$ & 46 & 52 & 42 & 48 & 88 & 100 & \\
\hline & Total & 55 & 56 & 44 & 44 & 99 & 100 & \\
\hline Relaciones sociales/trabajo en equipo & & $\mathbf{N}$ & $\%$ & $\mathbf{N}$ & $\%$ & $\mathbf{N}$ & $\%$ & p \\
\hline & No & 45 & 63 & 26 & 37 & 71 & 100 & \\
\hline Posición de responsabilidad en un grupo* & $S i$ & 10 & 36 & 18 & 64 & 28 & 100 & 0,01 \\
\hline & Total & 55 & 56 & 44 & 44 & 99 & 100 & \\
\hline Realización en el último año de alguna & No & 8 & 73 & 3 & 27 & 11 & 100 & \\
\hline actividad en equipo o grupo con los & $\mathrm{Si}$ & 48 & 54 & 41 & 46 & 89 & 100 & 0,23 \\
\hline compañeros de la escuela & Total & 56 & 56 & 44 & 44 & 100 & 100 & \\
\hline & No & 13 & 50 & 13 & 50 & 26 & 100 & \\
\hline $\begin{array}{l}\text { Relaciones sociales entre compañeros a través } \\
\text { de interacciones como ayuda o consejos }\end{array}$ & $S i$ & 43 & 58 & 31 & 42 & 74 & 100 & 0,47 \\
\hline & Total & 56 & 56 & 44 & 44 & 100 & 100 & \\
\hline
\end{tabular}


A partir de la intervención implementada se visualizan cambios estadísticamente significativos en el Gl en torno a la dimensión educación, donde los conocimientos adquiridos en el último año sobre agroecología pasaron de un $37 \%$ a un $63 \%$ (Tabla 1). Mientras que en el GC los alumnos reportan que estos conocimientos no fueron adquiridos, visualizando porcentajes similares en ambas instancias de relevamiento (46\% vs. $54 \%$ ).

En cuanto a la adquisición de conocimientos en el último año en el Gl sobre agroecología relacionada al ambiente o con otros dominios como es el caso del mercado de trabajo, el consumo de alimentos y/o nutrición y salud, también se observaron aumentos entre las etapas antes y después de la intervención $(p=0,0001)$. En este mismo grupo y en el marco de la dimensión alimentación saludable se constató un aumento en los conocimientos sobre alimentos agroecológicos (de 17\% a $83 \%$ ), siendo este cambio estadísticamente significativo ( $p=0,0001)$; además las creencias sobre los beneficios de los alimentos agroecológicos también aumentaron de manera significativa durante el periodo de intervención, pasando de un $15 \%$ a un $85 \%(p=0,0001)$ (Tabla 1$)$. No se encontraron cambios estadísticamente significativos en el GC.

En el Gl la incorporación de nuevos productos de origen vegetal a la alimentación se mantuvo de manera similar en el pre-test y post-test (51\% vs. $49 \%)$. Sin embargo, se observó un aumento significativo en el consumo del subgrupo de alimentos agroecológicos pasando de un $26 \%$ a un $74 \%$ $(p=0,0001)$ (Tabla 1). Mientras que en el GC el consumo de estos alimentos se tuvo un leve descenso (59\% y $41 \%$ ), no obstante no fue una diferencia estadísticamente significativa.

Al analizar la dimensión ambiente también se encontraron diferencias sobre las creencias de los adolescentes en torno a la relación entre producción de alimentos y cuidado del ambiente en el Gl donde aumentó del $32 \%$ al $68 \%(p=0,0001)$ (Tabla 1). Estas diferencias también se observaron en el GC, aunque los valores descendieron de un $70 \%$ a un $30 \%(p=0,0001)$.

En el Gl las categorías de creencias sobre la influencia de los factores ambientales en la salud y calidad de vida y la de realización de actividades sobre el cuidado del ambiente, se mantuvieron en porcentajes similares en ambas etapas.

Dentro de la dimensión relaciones sociales/trabajo en equipo, la categoría de posición de responsabilidad dentro de un grupo antes y después de la intervención en el Gl tuvo diferencias estadísticamente significativas ( $p=0,01$ ), pasando de un $36 \%$ a un $64 \%$ (Tabla 1 ), mientras que en el GC no se encontraron estas diferencias. La categoría de no realizar en el último año alguna actividad en equipo con los compañeros de la escuela bajó de $73 \%$ a $27 \%$ y las relaciones sociales a través de ayuda o consejo se mantuvieron constantes en las dos etapas de relevamiento (Tabla 1).

La frecuencia del consumo de frutas entre las etapas de pre-test y pos-test se mantuvo en valores similares en cuanto a la ingesta de un día a la semana (53\% y 47\%), aunque en el resto de categorías tendió a disminuir en ambos puntos temporales; esto se observó de manera similar en torno a la frecuencia del consumo de jugos de fruta natural en casi todas las categorías, aunque la categoría de un día a la semana paso del $40 \%$ al $60 \%$; mientras que en el consumo de verduras no feculentas la categoría de 1-3 días a la semana tuvo un aumento de un $43 \%$ a un $57 \%$, la categoría nunca bajó de un $67 \%$ a un $33 \%$; en el consumo de verduras feculentas se observó una disminución entre ambas instancias de relevamiento en casi todas las categorías en especial de 4-5 días a la semana (pasando de un $60 \%$ a un $40 \%$ ) y la de más de 5 días a la semana (65\% a un $35 \%$ ) (Tabla 2 ).

Tabla 2. Frecuencia de consumo de alimentos de origen vegetal antes y después de la intervención

\begin{tabular}{|l|lccccccc|}
\hline \multirow{2}{*}{ Frecuencia de consumo } & \multicolumn{3}{c}{ Pre-test } & \multicolumn{2}{c|}{ Pos-test } & \multicolumn{2}{c|}{ Total } \\
& & $\mathrm{N}$ & $\%$ & $\mathrm{~N}$ & $\%$ & $\mathrm{~N}$ & $\%$ \\
\hline \multirow{5}{*}{ Frutas } & Nunca & 0 & 0 & 2 & 100 & 2 & 100 \\
& 1 día & 8 & 53 & 7 & 47 & 15 & 100 \\
& $1-3$ días & 23 & 58 & 17 & 43 & 40 & 100 \\
& $4-5$ días & 9 & 64 & 5 & 36 & 14 & 100 \\
& Más de 5 días & 15 & 56 & 12 & 44 & 27 & 100 \\
\hline
\end{tabular}




\begin{tabular}{|c|c|c|c|c|c|c|c|}
\hline & \\
\hline & Total & 55 & 56 & 43 & 44 & 98 & 100 \\
\hline \multirow{6}{*}{ Jugos de fruta natural } & Nunca & 12 & 57 & 2 & 43 & 21 & 100 \\
\hline & 1 día & 10 & 40 & 15 & 60 & 25 & 100 \\
\hline & 1-3 días & 10 & 56 & 8 & 44 & 18 & 100 \\
\hline & 4-5 días & 9 & 75 & 3 & 25 & 12 & 100 \\
\hline & Más de 5 días & 11 & 61 & 7 & 39 & 18 & 100 \\
\hline & Total & 52 & 55 & 42 & 45 & 94 & 100 \\
\hline \multirow{6}{*}{ Verduras no feculentas } & Nunca & 4 & 67 & 2 & 33 & 6 & 100 \\
\hline & 1 día & 14 & 48 & 15 & 52 & 29 & 100 \\
\hline & 1-3 días & 10 & 43 & 13 & 57 & 23 & 100 \\
\hline & 4-5 días & 15 & 75 & 5 & 25 & 20 & 100 \\
\hline & Más de 5 días & 9 & 53 & 8 & 47 & 17 & 100 \\
\hline & Total & 52 & 55 & 43 & 45 & 95 & 100 \\
\hline \multirow{6}{*}{ Verduras feculentas } & Nunca & 4 & 36 & 7 & 64 & 11 & 100 \\
\hline & 1 día & 12 & 63 & 7 & 37 & 19 & 100 \\
\hline & 1-3 días & 10 & 48 & 11 & 52 & 21 & 100 \\
\hline & 4-5 días & 6 & 60 & 4 & 40 & 10 & 100 \\
\hline & Más de 5 días & 20 & 65 & 11 & 35 & 31 & 100 \\
\hline & Total & 52 & 57 & 40 & 43 & 92 & 100 \\
\hline
\end{tabular}

En cuanto la dimensión subjetiva de importancia personal en el Gl se visualizaron cambios marcados en la importancia de la alimentación, donde la categoría "importante" pasó de $45 \%$ en el pre-test al 55\% en el post-test. En cuanto a las relaciones personales con los compañeros la categoría "muy importante" e "importante" también se modificó levemente con una tendencia ascendente. En la importancia del ambiente se observaron algunos descensos, por ejemplo en la categoría "importante" pasó de $57 \%$ a $43 \%$, con valores similares en la importancia de la salud (Tabla 3 ).

Tabla 3. Importancia y satisfacción personal antes y después de la intervención

\begin{tabular}{|c|c|c|c|c|c|c|c|}
\hline \multirow{2}{*}{ Importancia personal } & & \multicolumn{2}{|c|}{ Pre-test } & \multicolumn{2}{|c|}{ Pos-test } & \multicolumn{2}{|c|}{ Total } \\
\hline & & $\mathrm{N}$ & $\%$ & $\mathrm{~N}$ & $\%$ & $\mathrm{~N}$ & $\%$ \\
\hline \multirow{6}{*}{ Importancia de la salud } & Muy importante & 51 & 57 & 39 & 43 & 90 & 100 \\
\hline & Importante & 4 & 57 & 3 & 43 & 7 & 100 \\
\hline & Algo importante & 0 & 0 & 1 & 100 & 1 & 100 \\
\hline & Levemente importante & 0 & 0 & 0 & 0 & 0 & 100 \\
\hline & No es importante & 0 & 0 & 0 & 0 & 0 & 100 \\
\hline & Total & 55 & 56 & 43 & 44 & 98 & 100 \\
\hline \multirow{6}{*}{ Importancia de la alimentación } & Muy importante & 41 & 61 & 26 & 39 & 67 & 100 \\
\hline & Importante & 13 & 45 & 16 & 55 & 29 & 100 \\
\hline & Algo importante & 1 & 50 & 1 & 50 & 2 & 100 \\
\hline & Levemente importante & 0 & 0 & 0 & 0 & 0 & 100 \\
\hline & No es importante & 0 & 0 & 0 & 0 & 0 & 100 \\
\hline & Total & 55 & 56 & 43 & 44 & 98 & 100 \\
\hline \multirow{5}{*}{ Importancia del ambiente } & Muy importante & 32 & 54 & 27 & 46 & 59 & 100 \\
\hline & Importante & 20 & 57 & 15 & 43 & 35 & 100 \\
\hline & Algo importante & 2 & 100 & 0 & 0 & 2 & 100 \\
\hline & Levemente importante & 1 & 50 & 1 & 50 & 2 & 100 \\
\hline & No es importante & 0 & 0 & 0 & 0 & 0 & 100 \\
\hline
\end{tabular}




\begin{tabular}{|c|c|c|c|c|c|c|c|}
\hline & Total & 55 & 56 & 43 & 44 & 98 & 100 \\
\hline \multirow{6}{*}{$\begin{array}{l}\text { Importancia de las relaciones estrechas con compañeros de la } \\
\text { escuela }\end{array}$} & Muy importante & 15 & 47 & 17 & 53 & 32 & 100 \\
\hline & Importante & 18 & 49 & 19 & 51 & 37 & 100 \\
\hline & Algo importante & 14 & 78 & 4 & 22 & 18 & 100 \\
\hline & Levemente importante & 5 & 63 & 3 & 38 & 8 & 100 \\
\hline & No es importante & 3 & 100 & 0 & 0 & 3 & 100 \\
\hline & Total & 55 & 56 & 43 & 44 & 98 & 100 \\
\hline \multirow{6}{*}{ Importancia del trabajo rentado } & Muy importante & 17 & 50 & 17 & 50 & 34 & 100 \\
\hline & Importante & 23 & 58 & 17 & 42 & 40 & 100 \\
\hline & Algo importante & 9 & 60 & 6 & 40 & 15 & 100 \\
\hline & Levemente importante & 2 & 50 & 2 & 50 & 4 & 100 \\
\hline & No es importante & 3 & 100 & 0 & 0 & 3 & 100 \\
\hline & Total & 54 & 56 & 42 & 44 & 96 & 100 \\
\hline \multirow{6}{*}{$\begin{array}{l}\text { Importancia de las actividades compartidas con compañeros de } \\
\text { la escuela }\end{array}$} & Muy importante & 13 & 76 & 4 & 24 & 17 & 100 \\
\hline & Importante & 19 & 51 & 18 & 49 & 37 & 100 \\
\hline & Algo importante & 17 & 50 & 17 & 50 & 34 & 100 \\
\hline & Levemente importante & 3 & 75 & 1 & 25 & 4 & 100 \\
\hline & No es importante & 3 & 43 & 4 & 57 & 7 & 100 \\
\hline & Total & 55 & 56 & 44 & 44 & 99 & 100 \\
\hline \multicolumn{8}{|l|}{ Satisfacción personal } \\
\hline \multirow{6}{*}{ Satisfacción con la salud } & Demasiado satisfecho & 30 & 60 & 20 & 40 & 50 & 100 \\
\hline & Satisfecho & 8 & 32 & 17 & 68 & 25 & 100 \\
\hline & Parcialmente satisfecho & 15 & 75 & 5 & 25 & 20 & 100 \\
\hline & Parcialmente insatisfecho & 1 & 50 & 1 & 50 & 2 & 100 \\
\hline & Insatisfecho & 1 & 50 & 1 & 50 & 2 & 100 \\
\hline & Total & 55 & 56 & 44 & 44 & 99 & 100 \\
\hline \multirow{6}{*}{ Satisfacción con la alimentación } & Demasiado satisfecho & 26 & 68 & 12 & 32 & 38 & 100 \\
\hline & Satisfecho & 8 & 24 & 25 & 76 & 33 & 100 \\
\hline & Parcialmente satisfecho & 16 & 80 & 4 & 20 & 20 & 100 \\
\hline & Parcialmente insatisfecho & 2 & 50 & 2 & 50 & 4 & 100 \\
\hline & Insatisfecho & 3 & 75 & 1 & 25 & 4 & 100 \\
\hline & Total & 55 & 56 & 44 & 44 & 99 & 100 \\
\hline \multirow{6}{*}{ Satisfacción con el ambiente } & Demasiado satisfecho & 11 & 65 & 6 & 35 & 17 & 100 \\
\hline & Satisfecho & 5 & 23 & 17 & 77 & 22 & 100 \\
\hline & Parcialmente satisfecho & 23 & 62 & 14 & 38 & 37 & 100 \\
\hline & Parcialmente insatisfecho & 10 & 77 & 3 & 23 & 13 & 100 \\
\hline & Insatisfecho & 6 & 60 & 4 & 40 & 10 & 100 \\
\hline & Total & 55 & 56 & 44 & 44 & 99 & 100 \\
\hline \multirow{6}{*}{ Satisfacción con las relaciones con los compañeros de la escuela } & Demasiado satisfecho & 6 & 43 & 8 & 57 & 14 & 100 \\
\hline & Satisfecho & 9 & 27 & 24 & 73 & 33 & 100 \\
\hline & Parcialmente satisfecho & 38 & 81 & 9 & 19 & 47 & 100 \\
\hline & Parcialmente insatisfecho & 0 & 0 & 2 & 100 & 2 & 100 \\
\hline & Insatisfecho & 2 & 67 & 1 & 33 & 3 & 100 \\
\hline & Total & 55 & 56 & 44 & 44 & 99 & 100 \\
\hline
\end{tabular}




\begin{tabular}{|l|lcccccc|}
\hline & Demasiado satisfecho & 7 & 41 & 10 & 59 & 17 & 100 \\
Satisfacción con las actividades que realiza con compañeros de & Satisfecho & 16 & 38 & 26 & 62 & 42 & 100 \\
la escuela & Parcialmente satisfecho & 29 & 81 & 7 & 19 & 36 & 100 \\
& Parcialmente insatisfecho & 2 & 100 & 0 & 0 & 2 & 100 \\
& Insatisfecho & 1 & 50 & 1 & 50 & 2 & 100 \\
\cline { 2 - 7 } & Total & 55 & 56 & 44 & 44 & 99 & 100 \\
\hline
\end{tabular}

Los valores fueron expresados como porcentajes para variables categóricas.

Al analizar la dimensión subjetiva de satisfacción personal de los adolescentes, se visualizan como datos más relevantes que la satisfacción con la salud en la categoría "satisfecho" pasó de un $32 \%$ a $68 \%$, al igual que la alimentación (de $24 \%$ a $76 \%$ ), el ambiente (de $23 \%$ a $77 \%$ ) y las relaciones con los compañeros (de $27 \%$ a un $73 \%$ ) en esta misma categoría. Dentro de la dimensión de actividades con los compañeros de la escuela la categoría "demasiado satisfecho" pasó de $41 \%$ a 59\% (Tabla 3).

\section{Discusión}

Según la evidencia de experiencias documentadas a nivel internacional se observó que implementar acciones integrales en el ámbito escolar desde una perspectiva alimentaria puede tener impactos positivos en diferentes dimensiones de la calidad de vida de los y las adolescentes ${ }^{(30,31)}$ donde esta premisa fue en parte constatada desde este estudio.

Dentro de estas estrategias la agroecología escolar puede tener impactos positivos en algunas de estas múltiples dimensiones, como es el caso de la alimentación y nutrición saludable, la seguridad alimentaria y la salud de los alumnos/as como lo postulan algunos estudios previos ${ }^{(32-36)}$; en esta línea los hallazgos en este estudio son concordantes con estos postulados, donde se observó que después de la intervención algunos aspectos alimentarios nutricionales mejoraron; esto se dio por ejemplo a un aumento en el consumo de alimentos agroecológicos, un aumento del consumo de verduras no feculentas y una disminución de verduras en el grupo de adolescentes, no obstante se pudo observar que el incorporar nuevos alimentos de origen vegetal a la alimentación no tuvo cambios significativos; esto puede deberse a que lograr cambios en los hábitos alimentarios requiere de un tiempo importante y de establecer acciones sostenidas en diferentes espacios que trascienden la esfera escolar.

Las prácticas agroecológicas en la escuela pueden tener impactos en torno a la educación ambiental, al ecosistema y a conservar y preservar la tierra ${ }^{(32,37-38)}$; siendo evidenciado en este estudio en torno a la sensibilización de los jóvenes con respecto al ambiente a través la implementación de agroecología escolar. Sin embargo, no fue un componente en los que se vieron demasiados cambios significativos desde los distintos indicadores, esto puede deberse a que la escuela bajo estudio ya venía realizando actividades con un mayor foco en la educación ambiental.

Otro aspecto sobresaliente de la estrategia es que constituye una herramienta socio-educativa interesante que permite potenciar el desarrollo individual y social; generando canales de intercambio de experiencias, conocimientos y saberes, que fomentan la socialización y el trabajo en equipo y otros aspectos personales subjetivos de los actores ${ }^{(5,39-42)}$. Estos aspectos en parte se pudieron constatar en el grupo de intervención con respecto a la posición de responsabilidad y realización de actividad en un grupo como también a través de relaciones sociales o a través de cambios positivos en la importancia y satisfacción personal de los adolescentes principalmente en torno a su la alimentación, relaciones personales, salud y ambiente.

Además, antecedentes científicos estarían indicando que la agroecología en la escuela permite añadir una dimensión práctica y teórica, que sirva para reforzar, relacionar y articular las diferentes materias curriculares convirtiendo a estrategia en un eje trasversal del proyecto educativo institucional ${ }^{(32,37)}$ promoviendo en los adolescentes un análisis más integral y significativo de saberes tanto conceptuales como procedimentales. Este hecho fue evidenciado por ejemplo, en que los jóvenes que formaron parte de la intervención reportaron cambios en los conocimientos teóricos/prácticos sobre agroecología adquiridos durante el año.

Sin embargo, también se sabe que la agricultura ecológica escolar puede tener efectos trasformadores en cuanto a una futura inserción laboral para los adolescentes desde un perspectiva de economía social y 
solidaria, contribuyendo con el desarrollo local y personal ${ }^{(32)}$, sin embargo esta dimensión no se pudo constatar desde los resultados de este estudio esto pudo deberse a que el proceso de establecer cambios en la inserción laboral, y poder sensibilizar a los jóvenes en micro-emprendimientos alimentarios llevaría un mayor tiempo de acompañamiento y sostenibilidad de acciones.

Si bien todos los alumnos estuvieron expuestos por lo menos una vez a la intervención explicada principalmente porque su ejecución se llevó a cabo dentro del programa curricular institucional, el estudio presentó la limitación de pérdidas del seguimiento de los escolares (tanto en el grupo intervención como control), debido principalmente a problemáticas del contexto socio-educativo como deserción escolar o ausentismo. Sin embargo esto es concordante con la realidad educativa de Argentina, como lo plantea Unicef (s/f), si bien hoy existe una amplia cobertura que asegura el acceso a la escolaridad, buena parte de los alumnos que ingresan al nivel secundario no logran terminar y/o experimentan situaciones que dificultan su progreso y que conllevan al abandono; por ejemplo entre 2009-2010, la tasa de repitencia a nivel del país fue 10,5\%, de abandono el 10,6\% y de sobre edad un 38,3\% (INDEC, 2009/2010).

Además como lo expresa Beltran (2003) existe una disociación entre la evidencia obtenida en estudios experimentales y la aplicación en el mundo real y donde tener pérdidas de seguimiento por factores contextuales se podría considerar aceptable y realista en este tipo de diseño más flexible.

Otra limitación radica en la diferencia entre las edades de los grupos de intervención y control y en la proporción de mujeres con respecto a los varones, explicadas claramente por el carácter de factibilidad del estudio. No obstante las diferencias en cuanto al género pueden ser concordantes con la realidad del nivel medio de Argentina que exhibe una "brecha de género invertida" (OCDE, 2013) es decir una mayor asistencia escolar de mujeres que de varones, que posiblemente obedece a que este último grupo suele insertarse más temprano en el mercado laboral. Por lo tanto la muestra estudiada representaría a la población real que está inserta el sistema de educación pública del país.

La principal fortaleza de este trabajo a partir de evaluar una intervención compleja es que se constituye en un insumo relevante en el campo de las políticas públicas en salud y educación y proporciona evidencia sobre un problema de investigación de escasos antecedentes documentados en el país y región.

\section{Conclusión}

La importancia de la toma de conciencia sobre la interacción entre ambiente, en este caso el escolar, con aspectos de la calidad de vida y la salud de las poblaciones desde un paradigma sostenible, ha sido motivo de preocupación y reflexión a nivel internacional desde hace varias décadas ${ }^{(43)}$.

Si bien desde este estudio se identificaron resultados positivos en algunas esferas subjetivas y objetivas de la calidad de vida relacionada a la salud en la adolescencia a partir de una intervención basada en la agroecología escolar y que fue implementada en el "mundo real", también se pudieron evidenciar indicadores que reflejaron la realidad soci-educativa de los adolescentes que afectan el proceso de implementación y que serán necesarios de considerar para pensar intervenciones exitosas. Se concluye que la intervención tiene potencial para ser replicada a otros contextos.

\section{Financiamiento}

El estudio fue financiado por la Comisión Nacional Salud Investiga, Ministerio de Salud de la Nación y aprobado por el Comité de Ética del Hospital Nacional de Clínicas (CIEIS-HNC) y el Consejo de Evaluación Ética de Investigación en Salud (CoEIS) de Córdoba.

\section{Agradecimientos}

Se agradece a todos los actores de la escuela IPEM 360, Villa El Libertador, Córdoba en especial a adolescentes, docentes, no docentes y directivo. Al Programa Pro-Huerta INTA, Córdoba por los recursos y su labor durante la implementación del proyecto y a la Escuela de Nutrición, FCM, UNC por el soporte institucional. 


\section{Bibliografía}

1. Fondo de Población de las Naciones Unidas. El Estado de la población mundial 2014. New York, USA: UNFPA; 2014.

2. Instituto Nacional de Estadísticas y Censos. Sistema de estadísticas sociodemográficas. Área educación. [Internet]. Buenos Aires: INDEC [citado 5 jul 2016]. Disponible en: http://www.indec.gov.ar/indicadores-sociodemograficos.asp

3. Fondo de las Naciones Unidas para la Infancia. Datos sobre Infancia en Argentina. 2013. [Internet]. Buenos Aires: UNICEF [citado 5 may 2016]. Disponible en: http://www.unicef.org/argentina/spanish/overview_11124.htm

4. Mieles MD, Acosta A. Calidad de vida y derechos de la infancia: un desafío presente. Rev Latinoamericana de Ciencias Sociales, Niñez y Juventud. [Internet]. 2012. [citado 18 sep 2016]; 10:205-217. Disponible en http://www.redalyc.org/articulo.oa?id=77323982012

5. Torres MR. Comunidad de Aprendizaje. Repensando lo educativo desde el desarrollo local y desde el aprendizaje. [Internet]. Simposio Internacional sobre Comunidades de Aprendizaje, Barcelona Forum 2004. Barcelona, España: Fundación Forum. [citado $11 \quad$ abr 2016]; 43:16-36. http://www.upch.edu.pe/rector/durs/images/Biblio/MarcoConceptual/ComunidadesAprendizajeDesarrollo/repensandoloeduca tivodesdeeldesarrollolocal.pdf

6. McAleese JD, Rankin LL. Garden-based nutrition education affects fruit and vegetable consumption in sixth-grade adolescents. J Am Diet Assoc. 2007;107(4):662-5.

7. Ratcliffe MM, Merrigan KA, Rogers BL, Goldberg JP. The effects of school garden experiences on middle school-aged students' knowledge, attitudes, and behaviors associated with vegetable consumption. Health Promot Pract. 2011;12(1):3643.

8. Tamiru D, Argaw A, Gerbaba M, Nigussie A, Ayana G, Belachew T. Improving dietary diversity of school adolescents through school based nutrition education and home gardening in Jimma Zone: Quasi-experimental design. Eat Behav. 2016;23:1806.

9. Utter J, Denny S, Dyson B. School gardens and adolescent nutrition and BMI: Results from a national, multilevel study. Prev Med. 2016;83:1-4.

10. Berezowitz CK, Bontrager Yoder AB, Schoeller DA. School Gardens Enhance Academic Performance and Dietary Outcomes in Children. J Sch Health. 2015;85(8):508-18.

11. Gatto NM, Martinez LC, Spruijt-Metz D, Davis JN. LA sprouts randomized controlled nutrition and gardening program reduces obesity and metabolic risk in Latino youth. Obesity (Silver Spring, Md). 2015;23(6):1244-51.

12. Wansink B, Hanks AS, Just DR. A plant to plate pilot: a cold-climate high school garden increased vegetable selection but also waste. Acta Paediatr (Oslo, Norway : 1992). 2015;104(8):823-6.

13. Barnidge EK, Hipp PR, Estlund A, Duggan K, Barnhart KJ, Brownson RC. Association between community garden participation and fruit and vegetable consumption in rural Missouri. Int J Behav Nutr Phys Act. 2013;10:128.

14. Gatto NM, Ventura EE, Cook LT, Gyllenhammer LE, Davis JN. LA Sprouts: a garden-based nutrition intervention pilot program influences motivation and preferences for fruits and vegetables in Latino youth. J Acad Nutr Diet. 2012;112(6):91320.

15. Evans A, Ranjit N, Rutledge R, Medina J, Jennings R, et al. Exposure to multiple components of a garden-based intervention for middle school students increases fruit and vegetable consumption. Health promot pract. 2012;13(5):608-16.

16. Somerset S, Markwell K. Impact of a school-based food garden on attitudes and identification skills regarding vegetables and fruit: a 12-month intervention trial. Public Health Nutr. 2009;12(2):214-21.

17. Hale J, Knapp C, Bardwell L, Buchenau M, Marshall J, et al. Connecting food environments and health through the relational nature of aesthetics: gaining insight through the community gardening experience. Soc Sci Med (1982). 2011;72(11):185363.

18. Van Lier LE, Utter J, Denny S, Lucassen M, Dyson B, Clark T. Home Gardening and the Health and Well-Being of Adolescents. Health Promot Pract. 2016;18(1): 34-43

19. Black JL, Velazquez CE, Ahmadi N, Chapman GE, Carten S, et al. Sustainability and public health nutrition at school: assessing the integration of healthy and environmentally sustainable food initiatives in Vancouver schools. Public Health Nutr. 2015;18(13):2379-91

20. Davis JN, Spaniol MR, Somerset S. Sustenance and sustainability: maximizing the impact of school gardens on health outcomes. Public Health Nutr. 2015;18(13):2358-67.

21. Chawla L, Keena K, Pevec I, Stanley E. Green schoolyards as havens from stress and resources for resilience in childhood and adolescence. Health \& place. 2014;28:1-13.

22. Ahmed AT, Oshiro CE, Loharuka S, Novotny R. Perceptions of middle school educators in Hawai'i about school-based gardening and child health. Hawaii Med J. 2011;70(7 Suppl 1):11-5.

23. Cummins RA. Comprehensive quality of life scale- School version. Australia: School of Psychology Deakin University; 1997.

24. Freyre P. Concientización. Buenos Aires: Ed. Búsqueda, 1974

25. Ausubel DP, Hanesian H, Novak JD. Psicología Educativa. Un punto de vista cognitivo. México: Trillas, 1976.

26. The World Health Organization Quality of Life Assessment (WHOQOL). Position Paper from the World Health Organization. Soc Sci Med. [Internet]. 1995. [citado 3 abr 2016]; 41:1403-9. Disponible en http://www.ncbi.nlm.nih.gov/pubmed/8560308

27. Baldi López G, García Quiroga E. Calidad de vida y medio ambiente. La psicología ambiental. Rev Universidades. [Internet]. 2005. [citado 23 sep 2016]; 30: 9-16. Disponible en: http://www.redalyc.org/articulo.oa?id=37303003

28. Palomba, R. Calidad de Vida: Conceptos y medidas. [Internet]. 2002; Roma, Italia: Institute of Population Research and Social Policies. [citado 11 abr 2016]. Disponible en: http://www.cepal.org/celade/agenda/2/10592/envejecimientorp1_ppt.pdf

29. Fernández Mayoralas G, Rojo Pérez F. Calidad de vida y salud: planteamientos conceptuales y métodos de investigación. $\begin{array}{llllll}\text { Territoris. } \quad \text { [Internet]. } 2005 . & \text { [citado } 21 & \text { sep 2016]; } & \text { 5:117-135. Disponible en: }\end{array}$ http://dialnet.unirioja.es/servlet/articulo?codigo=2139763

30. Food and Agriculture Organization of the United Nations. School Feeding and the Possibilities for Direct Purchases from Family Farming - Case Studies in Eight Countries. Santiago de Chile: FAO; 2013.

31. Food and Agriculture Organization, Brazilian Cooperation Agency. Strengthening School Meal Programmes under the Hunger Free Latin America and the Caribbean 2025 Initiative. Brazil: FAO/ABC; 2008. 
32. Organización de las Naciones Unidas para la Agricultura y la Alimentación. Nueva política de huertos escolares. Roma, Italia: FAO; 2010.

33. Robinson-O'Brien R, Story M, Heim S. Impact of garden-based youth nutrition intervention programs: A review. J Am Diet Assoc. [Internet]. 2009. [citado 18 sep 2016]; 109: 273-280. Disponible en: https://www.ncbi.nlm.nih.gov/pubmed/19167954

34. Heim S, Stang J, Ireland M. A garden pilot project enhances fruit and vegetable consumption among children. J Am Diet Assoc. [Internet]. 2009. [citado 4 ago 2016]; 109:1220-1226. Disponible en: https://www.ncbi.nlm.nih.gov/pubmed/19559139

35. Parmer SM, Salisbury-Glennon J, Shannon D, Struempler B. School gardens: An experiential learning approach for a nutrition education program to increase fruit and vegetable knowledge, preference and consumption among second-grade students. J Nutr Educ and Behav. [Internet]. 2009. [citado 18 sep 2016]; 41:212-217. Disponible en: https://www.ncbi.nlm.nih.gov/pubmed/19411056

36. Ozer EJ. The effects of school gardens on students and schools: Conceptualization and considerations for maximizing healthy developmente. Health Educ Behav. [Internet]. 2007. [citado 18 sep 2016]; 34:846-863. Disponible en: http://heb.sagepub.com/content/34/6/846.abstract

37. Instituto Nacional de Tecnología Agropecuaria. Las hurtas escolares como un espacio de aprendizaje y servicio solidario. Una aproximación desde las experiencias. Buenos Aires, Argentina: INTA; 2010.

38. Skelly SM, Bradley JC. The growing phenomenon of school gardens: Measuring their variation and their affect on students' sense of responsibility and attitudes toward science and the environment. Educ \& Communication. [Internet]. 2007. [citado 11 abr 2016]; 6:97-104. Disponible en: http://www.kohalacenter.org/HISGN/pdf/Thegrowingphenomofschoolgardens.pdf

39. Waliczek TM, Bradley JC, Zajicek JM. The effect of school gardens on children's interpersonal relationships and attitudes toward school. Horttechnology. [Internet]. 2001. [citado 2 abr 2016]; 11:466-468. Diponible en: http://horttech.ashspublications.org/content/11/3/466.full.pdf

40. Morgan PJ, Warren JM, Lubans D, Saunders K, Quick G, Collins CE. The impact of nutrition education with and without a school garden on knowledge, vegetable intake and preferences and quality of school life among primary-school students. Public Health Nutr [Internet]. 2010. [citado 18 sep 2016]; vol. 13, núm. 11, pp. 1931-1940.

41. Castellanos GA. Implementación de huertos escolares basada en el modelo de educación ambiental en el ámbito formal a través del enfoque transversal educativo. La Paz: umsa; 2003.

42. Skinner EA, Chi U. Intrinsic motivation and engagement as 'active ingredients' in garden-based education: Examining models and measures derived from self-determination. J Env Educ. [Internet]. 2012. [citado 11 abr 2016]; 43:16-36. Disponible en: https://www.pdx.edu/psy/sites/www.pdx.edu.psy/files/2012Skinner,\%20Chi,\%20\%26\%20the\%20LEAG_JEE_2012\%20copy $\% 20(1) . p d f$

43. Naciones Unidas. (2012). El futuro que queremos: documento final aprobado en Río+ 20. Disponible en: http://www.un.org/es/comun/docs/?symbol=A/RES/66/28

\section{Anexo I}

Instrumento de recolección de datos sobre calidad de vida relacionada a la salud de los adolescentes

Encuesta No:

Nombre y apellido:.

Edad:..........

Curso:..........

Estamos realizando la siguiente encuesta en el marco de un proyecto de intervención titulado "Agroecología en la escuela y calidad de vida relacionada a la salud de los adolescentes" desde la Escuela de Nutrición, Facultad de Ciencias Médicas, Universidad Nacional de Córdoba (UNC), que tiene como objetivo conocer información sobre algunos aspectos relacionados a su calidad de vida relacionada a la salud. No existen respuestas correctas o incorrectas. Usted resultó incluido en la muestra de adolescentes a encuestar donde su participación es voluntaria. La información que se recoja será estrictamente confidencial y anónima.

\begin{tabular}{|c|c|c|c|c|}
\hline \multicolumn{5}{|c|}{ DIMENSIÓN MATERIAL } \\
\hline 1. & Educación & SI & NO & \\
\hline 1.1 & $\begin{array}{l}\text { ¿Cree que en el último } \\
\text { año lectivo ha } \\
\text { incorporado } \\
\text { conocimientos teóricos } \\
\text { y/o prácticos sobre } \\
\text { producción de alimentos } \\
\text { en la escuela? }\end{array}$ & & & 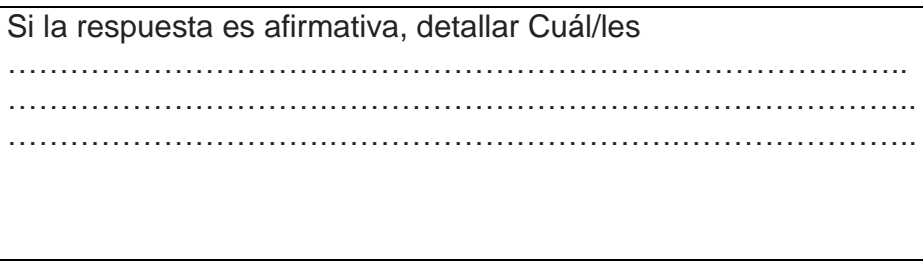 \\
\hline 1.2 & $\begin{array}{l}\text { ¿Cree que en el último } \\
\text { año lectivo ha } \\
\text { incorporado } \\
\text { conocimientos teóricos } \\
\text { y/o prácticos sobre } \\
\text { producción de alimentos } \\
\text { relacionada al medio } \\
\text { ambiente en la escuela? }\end{array}$ & & & 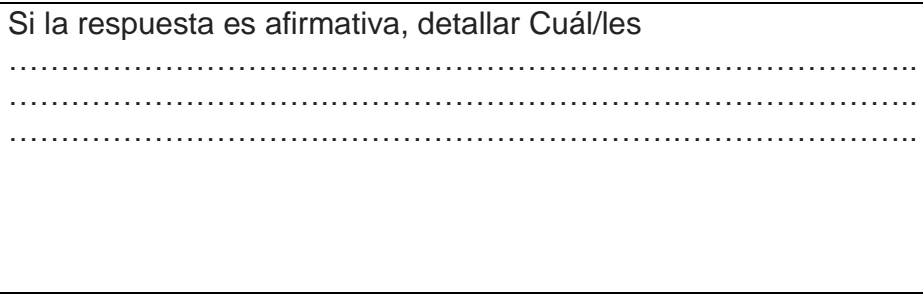 \\
\hline 1.3 & $\begin{array}{l}\text { ¿Cree que en el último } \\
\text { año lectivo ha } \\
\text { incorporado }\end{array}$ & & & Si la respuesta es afirmativa, detallar Cuál/les \\
\hline
\end{tabular}




\begin{tabular}{|c|c|c|c|c|c|c|}
\hline & $\begin{array}{l}\text { conocimientos teóricos } \\
\text { y/o prácticos sobre } \\
\text { producción de alimentos } \\
\text { relacionada al mercado } \\
\text { de trabajo en la escuela? }\end{array}$ & & & \multicolumn{3}{|c|}{ 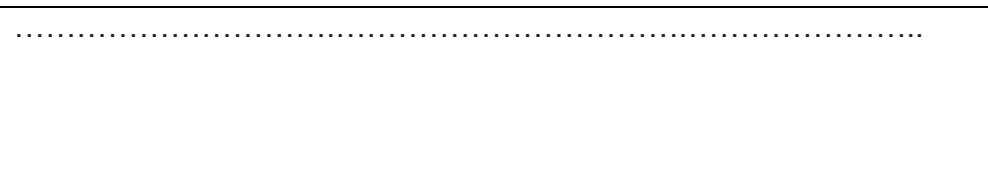 } \\
\hline 1.4 & $\begin{array}{l}\text { ¿Cree que en el último } \\
\text { año lectivo ha } \\
\text { incorporado } \\
\text { conocimientos teóricos } \\
\text { y/o prácticos sobre } \\
\text { producción de alimentos } \\
\text { relacionada al consumo } \\
\text { de alimentos en la } \\
\text { escuela? }\end{array}$ & & & \multicolumn{3}{|c|}{ Si la respuesta es afirmativa, detallar Cuál/les } \\
\hline 1.5 & $\begin{array}{l}\text { ¿Cree que en el último } \\
\text { año lectivo ha } \\
\text { incorporado } \\
\text { conocimientos teóricos } \\
\text { y/o prácticos sobre } \\
\text { producción de alimentos } \\
\text { relacionada a nuestro } \\
\text { estado de nutrición y } \\
\text { salud en la escuela? }\end{array}$ & & & \multicolumn{3}{|c|}{ Si la respuesta es afirmativa, detallar Cuál/les } \\
\hline 1.6 & $\begin{array}{l}\text { ¿Cree que en el último } \\
\text { año lectivo ha } \\
\text { incorporado } \\
\text { conocimientos teóricos } \\
\text { y/o prácticos sobre } \\
\text { producción de alimentos } \\
\text { relacionada a otros } \\
\text { aspectos en la escuela? }\end{array}$ & & & \multicolumn{3}{|c|}{ Si la respuesta es afirmativa, detallar Cuál/les } \\
\hline 2. & $\begin{array}{c}\text { Posición en el mercado } \\
\text { de trabajo }\end{array}$ & SI & NO & & & \\
\hline 2.1 & $\begin{array}{lr}\text { Actualmente, } & \text { ¿se } \\
\text { encuentra } & \text { realizando } \\
\text { alguna } & \text { actividad } \\
\text { rentada? } & \end{array}$ & & & & & \\
\hline 2.2 & $\begin{array}{l}\text { ¿Ha realizado alguna vez } \\
\text { alguna actividad laboral } \\
\text { en base a un micro- } \\
\text { emprendimiento? }\end{array}$ & & & $\begin{array}{l}\text { Si la respuesta es } \\
\text { afirmativa, } \\
\text { Cuál/les } \\
\ldots \ldots \ldots \ldots \ldots \ldots \\
\ldots \ldots \ldots \ldots \ldots\end{array}$ & 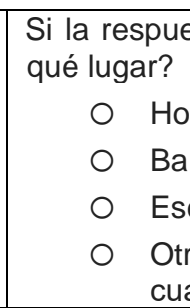 & $\begin{array}{l}\text { s afirmativa, ¿desde } \\
\text { specificar } \\
\text {........... }\end{array}$ \\
\hline 2.3 & $\begin{array}{l}\text { Si NO ha realizado nunca } \\
\text { un micro- } \\
\text { emprendimiento, } \\
\text { ¿tendría interés en } \\
\text { hacerlo en algún } \\
\text { momento orientado al } \\
\text { rubro producción y/o } \\
\text { comercialización de } \\
\text { alimentos teniendo en } \\
\text { cuenta aspectos } \\
\text { ecológicos? }\end{array}$ & & $\begin{array}{l}\mathrm{ML} \\
\text { Int } \\
\text { Alc } \\
\text { Le } \\
\text { No }\end{array}$ & $\begin{array}{l}\text { nteresado } \\
\text { sado } \\
\text { interesado } \\
\text { nente interesado } \\
\text { teresado }\end{array}$ & $\begin{array}{rr}\text { ¿Se } & \text { sier } \\
\text { implementa } \\
\bigcirc & \mathrm{Mu} \\
\bigcirc & \mathrm{Po} \\
\bigcirc & \mathrm{Ac} \\
\bigcirc & \mathrm{Ba} \\
\bigcirc & \mathrm{De}\end{array}$ & $\begin{array}{l}\text { capacitado para } \\
\text { le o normal }\end{array}$ \\
\hline 3. & Alimentación-nutrición & SI & NO & & & \\
\hline 3.1 & $\begin{array}{l}\text { ¿Incorporó en el último } \\
\text { año nuevas verduras y/o } \\
\text { frutas a su alimentación? }\end{array}$ & & & & & \\
\hline 3.2 & $\begin{array}{l}\text { ¿Conoce que son los } \\
\text { alimentos } \\
\text { agroecológicos? }\end{array}$ & & & 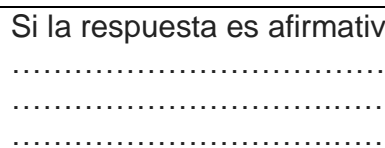 & $\begin{array}{l}\text { ite algún/os } \\
\ldots \ldots \ldots \ldots \ldots \ldots \\
\ldots \ldots \ldots \ldots \ldots \ldots \ldots \\
\ldots \ldots \ldots \ldots \ldots \ldots\end{array}$ & $\begin{array}{l}\ldots . \\
\cdots \cdots \\
\cdots \cdots\end{array}$ \\
\hline 3.3 & $\begin{array}{l}\text { Si conoce los alimentos } \\
\text { agroecológicos ¿Cree } \\
\text { que aportan beneficios a } \\
\text { su nutrición y salud? }\end{array}$ & & & 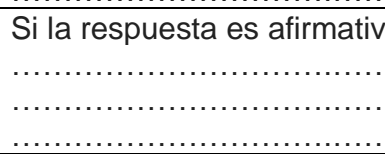 & $\begin{array}{l}\text { detallar Cuál/ } \\
\ldots \ldots \ldots \ldots \ldots \ldots \\
\ldots \ldots \ldots \ldots \ldots \ldots \ldots \\
\ldots \ldots \ldots \ldots \ldots \ldots\end{array}$ & 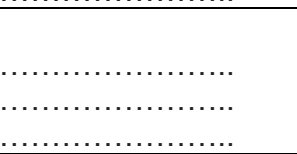 \\
\hline 3.4 & $\begin{array}{l}\text { ¿Consumió alguna vez } \\
\text { verduras y/o frutas } \\
\text { agroecológicas? }\end{array}$ & & & $\begin{array}{l}\text { Si la respuesta es } \\
\text { afirmativa, detallar } \\
\text { Cuál/les } \\
\ldots \ldots \ldots \ldots \ldots \ldots \ldots \ldots \\
\ldots \ldots \ldots \ldots \ldots \ldots \ldots \ldots\end{array}$ & $\begin{array}{l}\text { qué ámbito? } \\
\text { Hogar } \\
\text { Escuela } \\
\bigcirc \text { Otros, }\end{array}$ & $\begin{array}{l}\text { ¿Con que } \\
\text { frecuencia? } \\
\text { O Solo pocas } \\
\text { veces }\end{array}$ \\
\hline
\end{tabular}




\begin{tabular}{|c|c|c|c|c|c|c|c|}
\hline & & 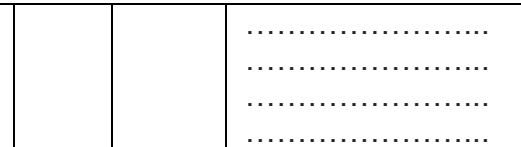 & & $\begin{array}{l}\text { speci } \\
\text { ual }\end{array}$ & & & $\begin{array}{l}\text { ria } \\
\text { nanal } \\
\text { isual }\end{array}$ \\
\hline \multirow{5}{*}{3.5} & \multirow{5}{*}{$\begin{array}{lr}\text { ¿Con que frecuencia } \\
\text { a la remana } \\
\text { consume r los } \\
\text { siguientes } \\
\text { alimentos? } \\
\text { (marcar con una X) }\end{array}$} & Alimento & Nunca & $\begin{array}{c}1 \\
\text { día }\end{array}$ & $\begin{array}{c}\text { De } \\
1-3 \\
\text { días }\end{array}$ & $\begin{array}{c}\text { De } \\
3-5 \\
\text { días }\end{array}$ & \begin{tabular}{|c} 
Más \\
de \\
5 \\
días \\
\end{tabular} \\
\hline & & Futas & & & & & \\
\hline & & Jugo de fruta natural & & & & & \\
\hline & & $\begin{array}{l}\text { Verduras: papa, batata, mandioca, } \\
\text { choclo }\end{array}$ & & & & & \\
\hline & & $\begin{array}{l}\text { Verduras: otras diferentes a las } \\
\text { anteriores }\end{array}$ & & & & & \\
\hline
\end{tabular}

\begin{tabular}{|c|c|c|c|c|c|}
\hline \multicolumn{6}{|c|}{ DIMENSIÓN AMBIENTAL } \\
\hline 1. & $\begin{array}{c}\text { Aspectos del entorno } \\
\text { escolar } \\
\text { ambientalmente } \\
\text { saludables y } \\
\text { sostenibles }\end{array}$ & SI & NO & & \\
\hline 1.1 & $\begin{array}{l}\text { ¿Considera importante el } \\
\text { cuidado del ambiente en } \\
\text { donde vive? }\end{array}$ & & & \multicolumn{2}{|c|}{$\begin{array}{l}\text { Si la respuesta es afirmativa, marque el nivel de importancia que le } \\
\text { asigna: } \\
\text { O Muy poco } \\
\bigcirc \text { Poco } \\
\bigcirc \text { Aceptable } \\
\bigcirc \text { Mucho } \\
\bigcirc \text { Demasiado }\end{array}$} \\
\hline 1.2 & $\begin{array}{l}\text { ¿Considera importante } \\
\text { mejorar su ambiente } \\
\text { escolar? }\end{array}$ & & & $\begin{array}{l}\text { Si la respuesta es afirmativa, } \\
\text { marque el nivel de importancia } \\
\text { que le asigna: } \\
\bigcirc \text { Muy poco } \\
\bigcirc \text { Poco } \\
\bigcirc \text { Aceptable } \\
\bigcirc \text { Mucho } \\
\bigcirc \text { Demasiado }\end{array}$ & $\begin{array}{l}\text { Si la respuesta es afirmativa, } \\
\text { detalle que estrategias y/o } \\
\text { actividades implementaría para } \\
\text { mejorar su ambiente escolar: }\end{array}$ \\
\hline 1.3 & $\begin{array}{l}\text { ¿Cree que hay factores } \\
\text { de su ambiente que } \\
\text { influyen en su salud y } \\
\text { calidad de vida? }\end{array}$ & & & \multicolumn{2}{|c|}{$\begin{array}{l}\text { Si la respuesta es afirmativa, marque cual/es identifica que } \\
\text { influyen: } \\
\bigcirc \text { Físicos (temperatura, ruidos, radiaciones, etc.) } \\
\bigcirc \text { Químicos (contaminación por plaguicidas, metales pesados, } \\
\text { etc.) } \\
\bigcirc \text { Biológicos (presencia de bacterias, virus y otros } \\
\text { microorganismos patógenos) } \\
\bigcirc \text { Psico-sociales (estrés, consumo de sustancias, etc.) } \\
\bigcirc \text { Sociales y culturales (educación, trabajo, hábitos } \\
\text { alimentarios y de vida, violencia, inseguridad, etc.) }\end{array}$} \\
\hline 1.4 & $\begin{array}{l}\text { ¿Reconoce la presencia } \\
\text { de alguna forma de } \\
\text { contaminación dentro del } \\
\text { entorno donde vive, } \\
\text { trabaja, estudia, etc.? }\end{array}$ & & & \begin{tabular}{l} 
Si la respuesta es afirmativa, \\
detallar Cuál/les \\
\hdashline$\ldots \ldots \ldots \ldots \ldots \ldots \ldots \ldots \ldots \ldots \ldots \ldots \ldots$
\end{tabular} & $\begin{array}{l}\text { Si la respuesta es afirmativa, } \\
\text { ¿En qué espacio se encuentra } \\
\text { esa contaminación? } \\
\bigcirc \text { Hogar } \\
\bigcirc \text { Barrio } \\
\bigcirc \text { Escuela } \\
\bigcirc \text { Otros, especificar cual }\end{array}$ \\
\hline 1.5 & $\begin{array}{l}\text { ¿Ha realizado alguna vez } \\
\text { alguna actividad } \\
\text { relacionada a mejorar y/o } \\
\text { cuidar el ambiente en } \\
\text { donde se encuentra? }\end{array}$ & & & $\begin{array}{l}\text { Si la respuesta es afirmativa, } \\
\text { detallar Cuál/les } \\
\ldots \ldots \ldots \ldots \ldots \ldots \ldots \ldots \ldots \ldots \ldots\end{array}$ & 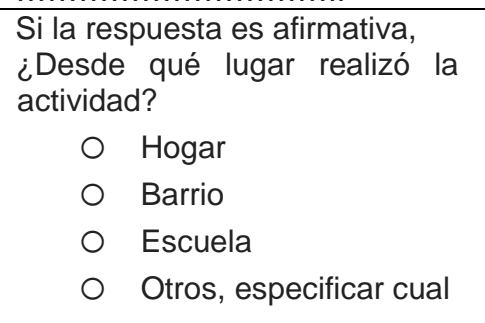 \\
\hline 1.6 & $\begin{array}{l}\text { ¿Cree que la producción } \\
\text { de alimentos se relaciona } \\
\text { con el cuidado del }\end{array}$ & & & \multicolumn{2}{|c|}{ Si la respuesta es afirmativa, fundamente porque } \\
\hline
\end{tabular}




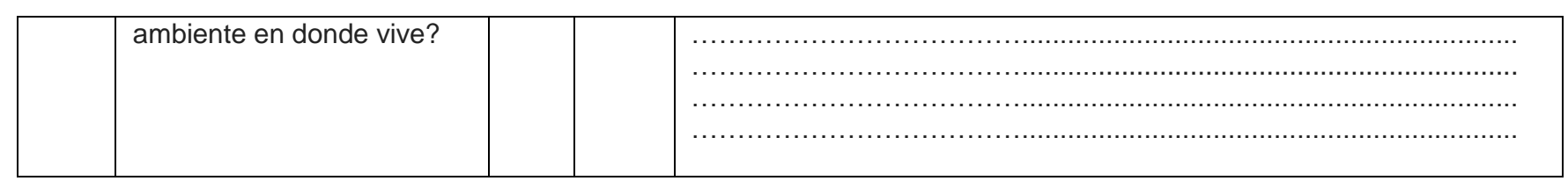

DIMENSIÓN RELACIONAMIENTO

\begin{tabular}{|c|c|c|c|c|}
\hline 1. & Relaciones sociales/trabajo en equipo & SI & NO & \\
\hline 1.1 & $\begin{array}{l}\text { ¿Ha realizado en el último año alguna actividad práctica } \\
\text { en equipo o grupo con los compañeros dentro de la } \\
\text { escuela? }\end{array}$ & & & $\begin{array}{l}\text { Si es afirmativa tu respuesta, con } \\
\text { qué frecuencia las realizaste: } \\
\bigcirc \text { Diaria } \\
\bigcirc \text { Varias veces a la semana } \\
\bigcirc \text { Una vez por semana } \\
\bigcirc \text { Una vez al mes } \\
\bigcirc \text { Menos de una vez al mes }\end{array}$ \\
\hline 1.2 & $\begin{array}{l}\text { ¿Tus compañeros de la escuela te piden ayuda o } \\
\text { consejo? }\end{array}$ & & & $\begin{array}{l}\text { Si es afirmativa tu respuesta, con } \\
\text { qué frecuencia: } \\
\bigcirc \text { Casi todos los días } \\
\bigcirc \text { Muy a menudo } \\
\bigcirc \text { A veces } \\
\bigcirc \text { No muy a menudo } \\
\bigcirc \text { Casi nunca } \\
\end{array}$ \\
\hline 1.2 & $\begin{array}{l}\text { En el último año, ¿ha tenido usted alguna posición de } \\
\text { responsabilidad en un equipo o grupo dentro de la } \\
\text { escuela? }\end{array}$ & & & $\begin{array}{l}\text { Si es afirmativo que posición ha } \\
\text { tenido: } \\
\bigcirc \text { Presidente / Coordinador } \\
\bigcirc \text { Tesorero / Secretario u otro } \\
\bigcirc \text { Miembro activo dentro del } \\
\text { equipo }\end{array}$ \\
\hline 1.5 & $\begin{array}{l}\text { A continuación se muestra una lista de actividades. } \\
\text { Indicar cuantos días al mes realiza las siguientes } \\
\text { actividades }\end{array}$ & $\begin{array}{l}0 \\
0 \\
0 \\
0 \\
0 \\
0 \\
0 \\
0 \\
0 \\
0 \\
0 \\
0\end{array}$ & $\begin{array}{l}\text { Ir a un } \\
\text { Activida } \\
\text { escuela } \\
\text { Activida } \\
\text { escuela } \\
\text { Activida } \\
\text { Ir a eve } \\
\text { Ir a un I } \\
\text { Estar er } \\
\text { Salir a } \\
\text { Visitar a } \\
\text { Hacer o } \\
\text { Realiza } \\
\text { compra } \\
\text { Realiza }\end{array}$ & $\begin{array}{l}\text { b / grupo /ONG } \\
\text { s con sus compañeros DENTRO de la } \\
\text { s con sus compañeros FUERA de la } \\
\text { os deportivos } \\
\text { ar religioso } \\
\text { as redes sociales } \\
\text { mer fuera del hogar } \\
\text { amiliares } \\
\text { ortes } \\
\text { de alividades dentro del hogar (ej. } \\
\text { tras actividades (describa).............. }\end{array}$ \\
\hline 2. & \multicolumn{4}{|c|}{ Importancia personal } \\
\hline 2.1 & $\begin{array}{l}\text { ¿Qué tan importante SON para usted LAS COSAS } \\
\text { MATERIALES que posee? }\end{array}$ & & $\begin{array}{ll}O & \mathrm{Mu} \\
\mathrm{O} & \mathrm{Imr} \\
\mathrm{O} & \text { Alg } \\
\mathrm{O} & \text { LeI } \\
\mathrm{O} & \text { No }\end{array}$ & $\begin{array}{l}\text { mportante } \\
\text { rtante } \\
\text { importante } \\
\text { mente importante } \\
\text { s importante }\end{array}$ \\
\hline 2.2 & ¿Qué tan importante es para ustedes SU SALUD? & & $\begin{array}{ll}\bigcirc & \mathrm{Mu} \\
\bigcirc & \mathrm{Imr} \\
\mathrm{O} & \text { Alg } \\
\mathrm{O} & \text { LeI } \\
\bigcirc & \text { No }\end{array}$ & $\begin{array}{l}\text { mportante } \\
\text { rtante } \\
\text { importante } \\
\text { mente importante } \\
\text { s importante }\end{array}$ \\
\hline 2.3 & ¿Qué tan importante es para ustedes SU & & $\mathrm{O} \mathrm{Mu}$ & importante \\
\hline
\end{tabular}




\begin{tabular}{|c|c|c|c|}
\hline & ALIMENTACIÓN? & $\begin{array}{l}0 \\
0 \\
0 \\
0\end{array}$ & $\begin{array}{l}\text { Importante } \\
\text { Algo importante } \\
\text { Levemente importante } \\
\text { No es importante }\end{array}$ \\
\hline 2.4 & $\begin{array}{l}\text { ¿Qué tan importante es para ustedes EL MEDIO } \\
\text { AMBIENTE en donde vive? }\end{array}$ & $\begin{array}{l}0 \\
0 \\
0 \\
0 \\
0\end{array}$ & $\begin{array}{l}\text { Muy importante } \\
\text { Importante } \\
\text { Algo importante } \\
\text { Levemente importante } \\
\text { No es importante }\end{array}$ \\
\hline 2.5 & $\begin{array}{l}\text { ¿Qué tan importante es para ustedes EL TRABAJO } \\
\text { RENTADO? }\end{array}$ & $\begin{array}{l}0 \\
0 \\
0 \\
0 \\
0\end{array}$ & $\begin{array}{l}\text { Muy importante } \\
\text { Importante } \\
\text { Algo importante } \\
\text { Levemente importante } \\
\text { No es importante }\end{array}$ \\
\hline 2.6 & $\begin{array}{l}\text { ¿Qué tan importante es para usted LAS RELACIONES } \\
\text { PERSONALES CON SUS COMPAÑEROS en la } \\
\text { escuela? }\end{array}$ & $\begin{array}{l}0 \\
0 \\
0 \\
0 \\
0\end{array}$ & $\begin{array}{l}\text { Muy importante } \\
\text { Importante } \\
\text { Algo importante } \\
\text { Levemente importante } \\
\text { No es importante } \\
\end{array}$ \\
\hline 2.7 & $\begin{array}{l}\text { ¿Qué tan importante es usted HACER ACTIVIDADES } \\
\text { CON SUS COMPAÑEROS en la escuela? }\end{array}$ & $\begin{array}{l}0 \\
0 \\
0 \\
0 \\
0\end{array}$ & $\begin{array}{l}\text { Muy importante } \\
\text { Importante } \\
\text { Algo importante } \\
\text { Levemente importante } \\
\text { No es importante } \\
\end{array}$ \\
\hline 3. & Satisfacciór & & \\
\hline 3.1 & $\begin{array}{l}\text { ¿Qué tan satisfecho está usted con LAS COSAS } \\
\text { MATERIALES que posee? }\end{array}$ & $\begin{array}{l}0 \\
0 \\
0 \\
0 \\
0\end{array}$ & $\begin{array}{l}\text { Demasiado satisfecho } \\
\text { Satisfecho } \\
\text { Parcialmente satisfecho } \\
\text { Parcialmente insatisfecho } \\
\text { Insatisfecho }\end{array}$ \\
\hline 3.2 & ¿Qué tan satisfecho está usted con SU SALUD? & $\begin{array}{l}0 \\
0 \\
0 \\
0 \\
0\end{array}$ & $\begin{array}{l}\text { Demasiado satisfecho } \\
\text { Satisfecho } \\
\text { Parcialmente satisfecho } \\
\text { Parcialmente insatisfecho } \\
\text { Insatisfecho }\end{array}$ \\
\hline 3.3 & ¿Qué tan satisfecho esta con SU ALIMENTACIÓN? & $\begin{array}{l}0 \\
0 \\
0 \\
0 \\
0\end{array}$ & $\begin{array}{l}\text { Demasiado satisfecho } \\
\text { Satisfecho } \\
\text { Parcialmente satisfecho } \\
\text { Parcialmente insatisfecho } \\
\text { Insatisfecho }\end{array}$ \\
\hline 3.4 & $\begin{array}{l}\text { ¿Qué tan satisfecho esta con EL MEDIO AMBIENTE en } \\
\text { donde vive? }\end{array}$ & $\begin{array}{l}0 \\
0 \\
0 \\
0 \\
0\end{array}$ & $\begin{array}{l}\text { Demasiado satisfecho } \\
\text { Satisfecho } \\
\text { Parcialmente satisfecho } \\
\text { Parcialmente insatisfecho } \\
\text { Insatisfecho }\end{array}$ \\
\hline 3.5 & $\begin{array}{l}\text { ¿Qué tan satisfecho está usted con SUS RELACIONES } \\
\text { PERSONALES CON SUS COMPAÑEROS en la } \\
\text { escuela? }\end{array}$ & $\begin{array}{l}0 \\
0 \\
0 \\
0 \\
0\end{array}$ & $\begin{array}{l}\text { Demasiado satisfecho } \\
\text { Satisfecho } \\
\text { Parcialmente satisfecho } \\
\text { Parcialmente insatisfecho } \\
\text { Insatisfecho }\end{array}$ \\
\hline
\end{tabular}


